\title{
AN IMPLICIT FUNCTION THEOREM WITHOUT DIFFERENTIABILITY ${ }^{1}$
}

\author{
J. WARGA
}

\begin{abstract}
We combine a "global" version of the classical inverse function theorem with Schauder's fixed point theorem to investigate the existence and continuity properties of a function $(F, x) \rightarrow \eta(F, x)$ such that $\eta(F, x)=$ $F(\eta(F, x), x)$.
\end{abstract}

Let $\mathcal{Y}$ be a Banach space, $Y, K \subset \mathcal{Y}, Y$ open, $K$ compact, $X$ a Hausdorff space, and $F: Y \times X \rightarrow K$ a continuous function. There are two classical theorems that ensure the existence of some set $\tilde{X} \subset X$ and of an implicit function $\eta: \tilde{X} \rightarrow Y$ such that

$$
\eta(x)=F(\eta(x), x) \quad(x \in \tilde{X}),
$$

namely the implicit function theorem (IFT) and Schauder's fixed point theorem. We shall combine a "global" variant of IFT with Schauder's theorem to investigate the existence and continuity of a function $(F, x) \rightarrow$ $\eta(F, x)$ such that $\eta(F, x)=F(\eta(F, x), x)$ for $x \in X$ and for continuous $F$ : $Y \times X \rightarrow K$ that are sufficiently "close" to some $H: Y \rightarrow K$ with a fixed point $y_{0}$.

This last problem arose in the study of the controllability at a control $u_{0}$ of the functional-integral equation

$$
y(t)=\int f_{0}(t, \tau, \xi(y)(\tau), u(\tau)) \mu(d \tau) \quad(t \in T),
$$

where $T$ is a compact metric space, $\mu$ a positive nonatomic Radon measure on $T, y \in C\left(T, \mathbf{R}^{n}\right), u$ a control function, and $\xi$ a "p-hereditary" [4, p. 203] transformation from $C\left(T, \mathbf{R}^{n}\right)$ to $L^{\infty}\left(\mu, \mathbf{R}^{k}\right)$. This equation, in which $f_{0}$ is Lipschitz continuous (but not necessarily differentiable) with respect to its third argument, is investigated by approximating $f_{0}$ uniformly with appropriate functions $f_{i}$ which are $C^{1}$ in that argument.

Our present results are summarized in Lemma 1 and in Theorem 1 (which generalizes [5, Theorem 3.1, p. 20]). We write $I$ for the identity mapping in $\mathscr{Y}$, $S(a, \alpha)\left[S^{F}(a, \alpha)\right]$ for the open [closed] ball in $\mathcal{Y}$ of center $a$ and radius $\alpha$,

Received by the editors June 7, 1977.

AMS (MOS) subject classifications (1970). Primary 26A57, 47H15; Secondary 26A96, 47H10, 54C65.

Key words and phrases. Implicit functions, nondifferentiable functions, fixed points, Banach spaces, measurable and continuous selections.

'Partially supported by Grant MCS 76-06756 of the National Science Foundation.

(c) American Mathematical Society 1978 
$d[A, y]$ for the distance between a point $y$ and a set $A, \stackrel{\Delta}{=}$ for "equal by definition", and

$$
S(A, \alpha) \stackrel{\Delta}{=}\{y \in \mathcal{Y} \mid d[A, y]<\alpha\} .
$$

THEOREM 1. Let $\mathcal{Y}$ be a Banach space, $Y$ an open subset of $\mathcal{Y}, K$ a compact subset of $\mathcal{Y}$, and $X$ a Hausdorff space. Suppose that $0<\alpha, c<\infty, S^{F}\left(y_{0}, \alpha\right)$ $\subset Y, G_{0}: Y \rightarrow K$ is $C^{1}$, and

$$
\left|\left[I-G_{0}^{\prime}(y)\right]^{-1}\right| \leqslant c \quad(y \in Y) .
$$

Let $\tilde{K} \triangleq K \cap S^{F}\left(y_{0}, \alpha\right)$, and let $\mathcal{F}$ be the metric space of all continuous functions $F: Y \times X \rightarrow K$ such that

$$
\left|F(y, x)-G_{0}(y)+G_{0}\left(y_{0}\right)-y_{0}\right| \leqslant \alpha / c \quad\left[x \in X, y \in S^{F}\left(y_{0}, \alpha\right)\right],
$$

with two elements of $\mathcal{F}$ identified if they coincide on $\tilde{K} \times X$, and with the metric

$$
\rho\left(F, F_{1}\right) \stackrel{\Delta}{=} \sup \left\{\left|F(y, x)-F_{1}(y, x)\right| \mid(y, x) \in \tilde{K} \times X\right\} .
$$

Then

(I) For each $(F, x) \in \mathscr{F} \times X$, the equation $y=F(y, x)$ has a solution $y \in \tilde{K}$;

(II) There exists a Borel measurable function $\eta: \mathscr{F} \times X \rightarrow \tilde{K}$ such that

$$
\eta(F, x)=F(\eta(F, x), x) \quad(F \in \mathscr{F}, x \in X)
$$

(III) If $\bar{F} \in \mathcal{F}, \bar{x} \in X$ and if $\Phi(F, x) \triangleq\{y \in \tilde{K} \mid y=F(y, x)\}$ is a singleton at $(\bar{F}, \bar{x})$ then every selection $\tilde{\eta}$ of the set-valued mapping $\Phi$ is continuous at $(\bar{F}, \bar{x})$;

(IV) If $X$ is compact, $F \in \mathscr{F}$, and $\Phi(F, x)$ is the singleton $\{\eta(F, x)\}$ for each $x \in X$, then $\eta(F, \cdot)$ is continuous and

$$
\lim _{F_{1} \rightarrow F} \tilde{\eta}\left(F_{1}, x\right)=\eta(F, x) \text { uniformly on } X
$$

for every selection $\tilde{\eta}$ of $\Phi$.

REMARK (ADDED IN PROOF). The following, more general, proposition can be proved exactly as statement (I): Let $G: Y \rightarrow \mathcal{Y}$ be $C^{1},\left|G^{\prime}(y)^{-1}\right| \leqslant c$ ( $y \in Y$ ), $G\left(y_{0}\right)=0$, and let $\Gamma: Y \rightarrow K$ be continuous and such that $|\Gamma(y)| \leqslant$ $\beta<\alpha / c(y \in Y)$. Then the equation $G(y)+\Gamma(y)=z$ has a solution $y \in$ $S^{F}\left(y_{0}, \alpha\right)$ for every $z \in \mathcal{Y}$ with $|z| \leqslant \alpha / c-\beta$.

In order to prove Theorem 1 we shall first require a "global" version of the classical inverse function theorem.

LEMMA 1. Let $Y$ be an open subset of a Banach space $\mathcal{Y}, 0<\alpha, c<\infty$, $S^{F}\left(y_{0}, \alpha\right) \subset Y$ and $G: Y \rightarrow \mathcal{Y}$ a $C^{1}$ function such that

$$
G\left(y_{0}\right)=0, \quad\left|G^{\prime}(y)^{-1}\right| \leqslant c \quad(y \in Y) .
$$

Then there exists a unique $C^{1}$ function $u: S^{F}(0, \alpha / c) \rightarrow S^{F}\left(y_{0}, \alpha\right)$ such that

$$
G(u(x))=x \quad(|x| \leqslant \alpha / c), \quad u(0)=y_{0} .
$$

Proof. We first recall that, by the classical inverse and implicit function 
theorems (as stated, e.g. in [2, pp. 265, 268]), for every point $\eta_{0} \in Y$ and a corresponding $x_{0} \stackrel{\Delta}{=} G\left(\eta_{0}\right)$, there exist $\varepsilon_{0}>0$ and a unique $C^{1}$ function $v_{0}$ : $S\left(x_{0}, \varepsilon_{0}\right) \rightarrow Y$ such that

$$
G\left(v_{0}(x)\right)=x \quad\left(\left|x-x_{0}\right|<\varepsilon_{0}\right), \quad v_{0}\left(x_{0}\right)=\eta_{0} .
$$

Furthermore, if $A$ is a connected open subset of $\mathscr{Y}, x_{0} \in A$, and $u_{1}: A \rightarrow Y$ and $u_{2}: A \rightarrow Y$ are two continuous functions such that

$$
G\left(u_{i}(x)\right)=x \quad(i=1,2, x \in A), \quad u_{1}\left(x_{0}\right)=u_{2}\left(x_{0}\right)
$$

then $u_{1}=u_{2}, u_{1}$ is $C^{1}$ and $u_{1}^{\prime}(x)=G^{\prime}\left(u_{1}(x)\right)^{-1},(x \in A)$. We shall henceforth refer to the above assertions as IFT.

Let $a \in \mathcal{Y}$ and $|a|=1$. We shall denote by $\mathscr{B}$ the collection of all points $\beta \in[0, \alpha / c]$ such that there exist $\varepsilon_{\beta}>0$, a corresponding open convex set $U_{\beta}=^{\Delta} S\left([0, \beta] a, \varepsilon_{\beta}\right)$ and a $C^{1}$ function $v_{\beta}: U_{\beta} \rightarrow Y$ satisfying

$$
G\left(v_{\beta}(x)\right)=x \quad\left(x \in U_{\beta}\right), \quad v_{\beta}(0)=y_{0} .
$$

It follows from IFT that $0 \in \mathscr{B}$ and it is clear that $\mathscr{B}$ is a relatively open subinterval of $[0, \alpha / c]$.

Now let $\bar{\beta} \triangleq$ sup $\mathscr{B}$ and $U \triangleq \cup_{\beta \in \mathscr{B}} U_{\beta}$. Then $U$ is an open and connected neighborhood of $\mathscr{B} a$ and, by IFT, $v_{\beta}(x)=v_{\gamma}(x)$ if $\beta, \gamma \in \mathscr{B}$ and $x \in U_{\beta} \cap$ $U_{\gamma}$. We may therefore define a unique $C^{1}$ function $v: U \rightarrow Y$ by

$$
v(x)=v_{\beta}(x) \quad\left(\beta \in \mathscr{B}, x \in U_{\beta}\right) .
$$

We have $\left|v^{\prime}(x)\right|=\left|G^{\prime}(v(x))^{-1}\right| \leqslant c$ and therefore

$$
|v(\beta a)-v(0)|=\left|v(\beta a)-y_{0}\right|<c \beta \leqslant \alpha \quad(\beta \in \mathscr{B}) .
$$

Thus $w \triangleq \lim \nu(\beta a),(\beta \rightarrow \bar{\beta}, \beta \in \mathscr{B})$ exists, $w \in S^{F}\left(y_{0}, \alpha\right) \subset Y$ and $G(w)=\bar{\beta} a$. Again, by IFT, there exists $\bar{\varepsilon}>0$ and a unique $C^{1}$ function $\bar{v}$ : $S(\bar{\beta} a, \bar{\varepsilon}) \rightarrow Y$ satisfying

$$
\begin{aligned}
G(\bar{v}(x)) & =x \quad(|x-\bar{\beta} a|<\bar{\varepsilon}), \quad \bar{v}(\bar{\beta} a)=w, \\
\bar{v}(x) & =v(x) \quad\left(\beta \in \mathscr{B}, x \in U_{\beta} \cap S(\bar{\beta} a, \bar{\varepsilon})\right) .
\end{aligned}
$$

Since $\beta^{\prime} \stackrel{\Delta}{=} \bar{\beta}-\frac{1}{2} \bar{\varepsilon} \in \mathscr{B}$, the function $v$ is defined on $A_{\beta^{\prime}} \triangleq S\left(\left[0, \beta^{\prime}\right] a, \varepsilon^{\prime}\right)$, where $\varepsilon^{\prime} \stackrel{\Delta}{=} \operatorname{Min}\left(\frac{1}{2} \bar{\varepsilon}, \varepsilon_{\beta^{\prime}}\right)>0$. We may therefore define a $C^{1}$ function $u^{a}$ by

$$
\begin{gathered}
U^{a} \triangleq S\left([0, \bar{\beta}] a, \varepsilon^{\prime}\right), \quad u^{a}(x)=v(x) \quad\left(x \in A_{\beta^{\prime}}\right), \\
u^{a}(x)=\bar{v}(x) \quad\left(x \in U^{a} \sim A_{\beta^{\prime}}\right),
\end{gathered}
$$

which shows that $\bar{\beta}=\sup \mathscr{B} \in \mathscr{B}$. Thus $\mathscr{B}$ is a nonempty, open and closed subset of $[0, \alpha / c]$; hence $\mathscr{B}=[0, \alpha / c]$.

We now conclude that for every $a \in \mathcal{Y}$ with $|a|=1$ there exist an open connected neighborhood $U^{a}$ of $[0, \alpha / c] a$ and a unique continuous function $v^{a}: U^{a} \rightarrow Y$ such that

$$
G\left(v^{a}(x)\right)=x \quad\left(x \in U^{a}\right), \quad v^{a}(0)=y_{0} .
$$

Furthermore, by IFT, $v^{a}(x)=v^{b}(x)$ if $x \in U^{a} \cap U^{b}$. We may therefore 
define a unique continuous $u: S^{F}(0, \alpha / c) \rightarrow Y$ by

$$
u(x)=v^{a}(x) \quad\left(a \in \mathcal{Y},|a|=1, x \in U^{a}\right),
$$

and it follows from IFT that $u$ is $C^{1}$ and $\left|u(x)-y_{0}\right|<c|x| \leqslant \alpha$ for all $x \in S^{F}(0, \alpha / c)$. Q.E.D.

Proof of Theorem 1. Let $H(y) \triangleq G_{0}(y)+y_{0}-G_{0}\left(y_{0}\right)$. Then, by Lemma 1 , there exists a unique $C^{1}$ function $u: S^{F}(0, \alpha / c) \rightarrow S^{F}\left(y_{0}, \alpha\right)$ such that

$$
(I-H)(u(v))=v \quad(|v| \leqslant \alpha / c), \quad u(0)=y_{0} .
$$

If $F \in \mathscr{F}$ and $x \in X$ then $|F(y, x)-H(y)| \leqslant \alpha / c\left[y \in S^{F}\left(y_{0}, \alpha\right)\right]$. Thus $y \rightarrow u(F(y, x)-H(y))$ is a continuous mapping of $S^{F}\left(y_{0}, \alpha\right)$ into itself. Furthermore, since $F(y, x)-H(y) \in K-K+G_{0}\left(y_{0}\right)-y_{0}$, this mapping carries $S^{F}\left(y_{0}, \alpha\right)$ into a compact set. Therefore, by Schauder's fixed point theorem, this mapping has a fixed point $\bar{\eta}$. In view of (1), we have

$$
\bar{\eta}=u(F(\bar{\eta}, x)-H(\bar{\eta}))=H(\bar{\eta})+F(\bar{\eta}, x)-H(\bar{\eta})=F(\bar{\eta}, x) .
$$

This proves statement (I).

Now let

$$
\begin{gathered}
\Phi(F, x) \stackrel{\Delta}{=}\{y \in \tilde{K} \mid y=F(y, x)\} \quad(F \in \mathscr{F}, x \in X), \\
\text { Graph }(\Phi) \stackrel{\Delta}{=}\{((F, x), y) \mid y \in \Phi(F, x)\} .
\end{gathered}
$$

Then, by statement (I), the set $\Phi(F, x)$ is nonempty for all $(F, x) \in \mathcal{F} \times X$, and it is easy to see that each $\Phi(F, x)$ is closed in the compact space $\tilde{K}$ and Graph $(\Phi)$ is closed in $(\mathscr{F} \times X) \times \tilde{K}$. It follows that

(i) the set-valued mapping $\Phi$ is Borel measurable, that is, for each closed $C \subset \tilde{K}$, the set $\{(F, x) \mid \Phi(F, x) \cap C \neq \varnothing\}$ is Borel measurable (and, in fact, closed); and

(ii) by a theorem of Berge [1, Corollary to Theorem 7, p. 112], for every $(\bar{F}, \bar{x}) \in \mathscr{F} \times X$ and every open subset $U$ of the space $\tilde{K}$, with $\Phi(\bar{F}, \bar{x}) \subset U$, there exists a neighborhood $V$ of $(\bar{F}, \bar{x})$ in $\mathcal{F} \times X$ such that $\Phi(F, x) \subset U$ for all $(F, x) \in V$.

By a known measurable selection theorem [3, Theorem 4.1, p. 867], it follows from (i) that $\Phi$ has a Borel measurable selection $(F, x) \rightarrow \eta(F, x)$, which proves statement (II). Statement (III) follows directly from (ii), and (IV) follows from (III). Q.E.D.

Acknowledgement. I wish to thank Professor Togo Nishiura for suggesting improvements in Theorem 1 and in the proof of statements (II)-(IV).

\section{REFERENCES}

1. C. Berge, Topological spaces, Macmillan, New York, 1963.

2. J. Dieudonné, Foundations of modern analysis, Academic Press, New York, 1960. 
3. D. H. Wagner, Survey of measurable selection theorems, SIAM J. Control Optimization 15 (1977), 859-903.

4. J. Warga, Optimal control of differential and functional equations, Academic Press, New York, 1972.

5. Derivate containers, inverse functions, and controllability, in Calculus of Variations and Optimal Control Theory, D. L. Russell, ed., Academic Press, New York, 1976.

Departmiant of Mathenatics, Northeastern UNIVERstTy, Boston, Massachusetts 02115 